\title{
Low LINC01272 predicts poor prognosis of non-small cell lung cancer and its biological function in tumor cells by inhibiting miR-1303
}

\author{
SUE ZHANG and JIELU ZHOU \\ Anesthesiology Department, Weifang People's Hospital, Weifang, Shandong 261041, P.R. China
}

Received April 1, 2021; Accepted June 24, 2021

DOI: $10.3892 / \mathrm{ol} .2021 .12913$

\begin{abstract}
Non-small cell lung cancer (NSCLC) is a malignant tumor associated with poor prognosis. The clinical value of long non-coding RNAs (lncRNAs) in the pathomechanism of various types of human malignancy has attracted increasing attention. The present study aimed to investigate the expression of LINC01272 in NSCLC and to determine its prognostic value and biological role. Tumor and adjacent non-tumor tissues from 108 patients with NSCLC and NSCLC cell lines were used in this study. The expression levels of LINC01272 and microRNA (miR)-1303 in tissues of patients and NSCLC cell lines were evaluated by reverse transcription quantitative PCR. The relationship between LINC01272 and the overall survival of patients with NSCLC was analyzed by Kaplan-Meier survival curve and log-rank test. Cox regression analysis confirmed the prognostic value of LINC01272 in patients with NSCLC. Cell Counting Kit-8 assay was used to evaluate the proliferation of NSCLC cells. The migration and invasion of NSCLC cells were determined using Transwell assays. The interaction between LINC01272 and miR-1303 in NSCLC was confirmed by dual-luciferase reporter assay. LINC01272 downregulation in NSCLC tissues was associated with worse overall survival in patients based on bioinformatics analysis. Furthermore, LINC01272 expression, which was decreased in NSCLC tumor tissues and NSCLC cells, was considered as an independent prognostic biomarker in NSCLC. In addition, LINC01272 overexpression inhibited NSCLC cell proliferation, migration and invasion. miR-1303 expression, which was increased in tumor tissues, was sponged by LINC01272 and negatively correlated with LINC01272 expression. miR-1303 expression reversed the inhibitory effects of LINC01272 on NSCLC cell function. In summary, the findings from this study suggested that LINC01272 expression, which was
\end{abstract}

Correspondence to: Dr Jielu Zhou, Anesthesiology Department, Weifang People's Hospital, 151 Guangwen Street, Weifang, Shandong 261041, P.R. China

E-mail: zhoujielu7105@163.com

Key words: LINC01272, microRNA-1303, prognosis, non-small cell lung cancer, proliferation, migration, invasion decreased in NSCLC tumor tissues and NSCLC cells, may be used as an independent prognostic biomarker for patients with NSCLC and that its overexpression may suppress NSCLC cell proliferation, migration and invasion by inhibiting miR-1303.

\section{Introduction}

According to the International Agency for Research on Cancer, lung cancer was considered as the most common type of cancer and the leading cause of cancer-associated mortality in 2018, accounting for $18.4 \%$ of cancer-related deaths worldwide (1). Based on the histological characteristics of tumor cells, lung cancer is mainly divided into two categories, small cell lung cancer (SCLC) and non-small cell lung cancer (NSCLC), and $>80 \%$ of lung cancers belong to the NSCLC category (2). Most cancer patients are diagnosed with advanced cancer at the time of medical treatment, and the five-year survival rate is $<18 \%$ (3). Studying the pathogenesis of NSCLC and exploring novel therapeutic targets that could be effective for NSCLC treatment is therefore crucial.

Long non-coding RNAs (lncRNAs) are defined as transcripts of $>200$ nucleotides in length (4). LncRNAs have attracted increasing attention in the recent years due to their important roles in the pathological mechanisms of malignant tumors (5). These functional lncRNAs have clinical significance and biological functions in various types of cancer, such as the role of MVIH in pancreatic ductal adenocarcinomas (6), PCTST in pancreatic cancer (7) and HOTAIR in gastric cancer (8). The expression of LINC01272 has been found to be abnormal in numerous diseases, such as gastric cancer (9), inflammatory bowel diseases (10) and unstable atherosclerotic plaque (11). In addition, LINC01272 was reported to have an important role in promoting gastric cancer progression (9). However, the role of LINC01272 remains unclear in NSCLC. The results from our preliminary bioinformatics analysis using data from The Cancer Genome Atlas (TCGA) database demonstrated that LINC01272 expression is significantly downregulated in NSCLC tumor, and that patients with lung cancer and with low LINC01272 expression have a significantly worse overall survival than patients with high LINC01272 expression. Exploring the clinical significance and function of LINC01272 expression in patients with NSCLC is therefore of great importance to further understand the role of LINC01272 and improve the treatment of NSCLC. 
MicroRNAs (miRNAs) are a class of non-coding RNA molecules of $\sim 22$ nucleotide in length that promote the degradation of target mRNAs or inhibit mRNA translation by recognizing and binding to the 3'-untranslated regions of target mRNAs (12). Previous studies have demonstrated that microRNAs (miRNAs) play important roles in cancer progression by promoting or inhibiting tumorigenesis $(13,14)$. It has been reported that miR-1303 plays a crucial role in various types of cancer $(15,16)$. For example, it was shown that increased miR-1303 expression in prostate cancer tissues and cell lines could promote the proliferation, migration and invasion of prostate cancer cells (15). Zhang et al (16) reported that miR-1303 expression is decreased in gastric cancer tissues and cell lines, promoting gastric cancer cell proliferation, migration and invasion. Furthermore, a previous study demonstrated that increased expression of miR-1303 in NSCLC tissues and cells could promote NSCLC cell proliferation, migration and invasion and might therefore serve as a potential prognostic biomarker for NSCLC (17). In addition, our preliminary bioinformatics analysis predicted the binding site of LINC01272 to miR-1303. We therefore hypothesized that LINC01272 may have a role in NSCLC by targeting miR-1303.

The present study aimed to analyze the expression level of LINC01272 in patients with NSCLC, evaluate the prognostic value of LINC01272 and analyze the role of LINC01272/miR-1303 axis in NSCLC.

\section{Materials and methods}

Patients and sample collection. The experimental procedures were approved by the Ethics Committee of Weifang People's Hospital (approval no. 011827) and patients provided signed informed consents prior to sampling. Tumor tissue samples and adjacent non-tumor tissue samples $(>5 \mathrm{~cm}$ from the tumor margin) were collected from 108 patients with NSCLC who underwent curative resection between July 2012 and June 2015 at Weifang People's Hospital. All tissue samples were histopathologically verified and stored in liquid nitrogen. Patients who had received any anti-tumor treatment prior to the surgery were excluded from the study. All patients received a 5-year follow-up with monthly telephone or face-to-face appointment for patient survival. The clinicopathological characteristics of patients are presented in Table I.

Cell culture. The four NSCLC cell lines SK-MES-1 (cat. no. TCHu110), A549 (cat. no. TCHu150), NCI-H460 (cat. no. TCHu205) and NCI-H522 (cat. no. MZ-1314) and the normal lung cell line NHBE-T (cat. no. MZ-2677) were purchased from The Cell Bank of Type Culture Collection of The Chinese Academy of Sciences. All cells were cultured in Dulbecco's modified Eagle medium (DMEM; Invitrogen; Thermo Fisher Scientific, Inc.) supplemented with $10 \%$ FBS (Invitrogen; Thermo Fisher Scientific, Inc.) and placed at $37^{\circ} \mathrm{C}$ in a humidified incubator containing $5 \% \mathrm{CO}_{2}$.

Cell transfection. The pcDNA3.1, pcDNA3.1-LINC01272 expression vector, miR-1303 mimic and mimic negative control (mimic NC) were synthesized from Shanghai GenePharma Co., Ltd.. Briefly, for the synthesis of pcDNA3.1-LINC01272, the PCR products of LINC01272 were firstly cloned into
pUM-T vector and transformed into Escherichia coli DH5 $\alpha$ to obtain LINC01272 clones. The recombinant expression vector pcDNA3.1-LINC01272 was generated by subcloning the obtained LINC01272 clones directly into pcDNA3.1 plasmid through digestion and ligation. Then, $30 \mathrm{nM}$ pcDNA3.1 and $30 \mathrm{nM}$ pcDNA3.1-LINC01272 were transfected into A549 and $\mathrm{H} 460$ cells, and $50 \mathrm{nM}$ miR-1303 mimic (5'-UUUAGAGAC GGGGUCUUGCUCU-3') and $50 \mathrm{nM}$ mimic NC (5'-UUC UCCGAACGUGUCACGU-3') were transfected into A549 cells using Lipofectamine 3000 (Invitrogen; Thermo Fisher Scientific, Inc.) according to the manufacturers' protocol. Cells were used for subsequent experiments following $48 \mathrm{~h}$ transfection at $37^{\circ} \mathrm{C}$.

Bioinformatics analysis. GEPIA 2.0 (http://gepia2. cancer-pku.cn/\#index) (18) was used to analyze data from TCGA database (https://cancergenome.nih.gov/) and compare the expression levels of LINC01272 in lung adenocarcinoma (LUAD) and lung squamous cell carcinoma (LUSC) with that of healthy controls. Kaplan-Meier plotter (http://www.kmplot.com/analysis/) (19) was used to analyze the data of the Gene Expression Omnibus (GEO; https://www.ncbi.nlm.nih.gov/geo/), the European Genome-Phenome Archive (EGA; https://ega-archive. org/datasets) and TCGA database and evaluate the association between LINC01272 expression and the survival prognosis in patients with lung cancer. The lncBase v. 2 of DIANA (http://carolina.imis.athena-innovation. gr/diana_tools/web/index.php?r=lncbasev2/index) was used to predict the binding sites of LINC01272 to potential target miRNAs.

$R N A$ extraction and reverse transcription quantitative (RT-q) $P C R$. TRIzol reagent (Invitrogen; Thermo Fisher Scientific, Inc.) was used to extract total RNA from tissue samples and NSCLC cells. The purity and concentration of the extracted RNA were evaluated by NanoDrop 2000 (Thermo Fisher Scientific, Inc.). Then, cDNA was synthesized using the Revert Aid First Strand cDNA Synthesis Kit (Thermo Fisher Scientific) according to the manufacturers' instructions.

The levels of LINC01272 and miR-1303 were measured using RT-qPCR, which was carried out using a SYBR Green PCR kit (Bio-Rad Laboratories, Inc.) and the Applied Biosystems 7900 Real-Time PCR system (Applied Biosystems; Thermo Fisher Scientific, Inc.). LINC01272 and miR-1303 expression were normalized to GAPDH and U6, respectively. The PCR reaction conditions were as follows: $95^{\circ} \mathrm{C}$ for $10 \mathrm{~min}$, followed by 39 cycles at $95^{\circ} \mathrm{C}$ for $10 \mathrm{sec}$ and $60^{\circ} \mathrm{C}$ for $30 \mathrm{sec}$. The sequences of the primers were as follows: LINC01272, forward 5'-TGTTCACTGCTGTACACCCA-3', reverse 5'-TGT GGAGAGGGGATTTCTGG-3'; GAPDH, forward 5'-TGT TCGTCATGGGTGTGAAC-3', reverse 5'-ATGGCATGGACT GTGGTCAT-3'; miR-1303, forward 5'-GCCGAGTTTAGA GACGGGGT-3', reverse 5'-CTCAACTGGTGTCGTGGA-3'; and U6, forward 5'-CTCGCTTCGGCAGCACA-3' and reverse 5'-AACGCTTCACGAATTTGCGT-3'. The relative expression levels were calculated using the $2^{-\Delta \Delta \mathrm{Cq}}$ method (20).

Cell proliferation assay. The proliferation of A549 and H460 cells was evaluated using a Cell Counting Kit-8 (CCK-8) assay 
Table I. Association between LINC01272 expression and the clinicopathological characteristics of patients with non-small cell lung cancer.

LINC01272 expression

Variable

Number, $n=108$

Low (n=58)

High $(\mathrm{n}=50)$

P-values

Age, years

$\leq 60$

39

20

0.704

$>60$

69

38

0.971

Female

23

Male

65

35

0.982

Smoking

No

21

37

0.021

Tumor size, $\mathrm{cm}$

69

23

35

Lymph node metastasis

$54 \quad 23 \quad 31$

No

Yes

Differentiation

$\begin{array}{lll}60 & 28 & 32\end{array}$

Poor

TNM stage

$\begin{array}{llll}\text { I-II } & 46 & 18 & 28 \\ \text { III } & 62 & 40 & 22\end{array}$

TNM, Tumor-Node-Metastasis.

A

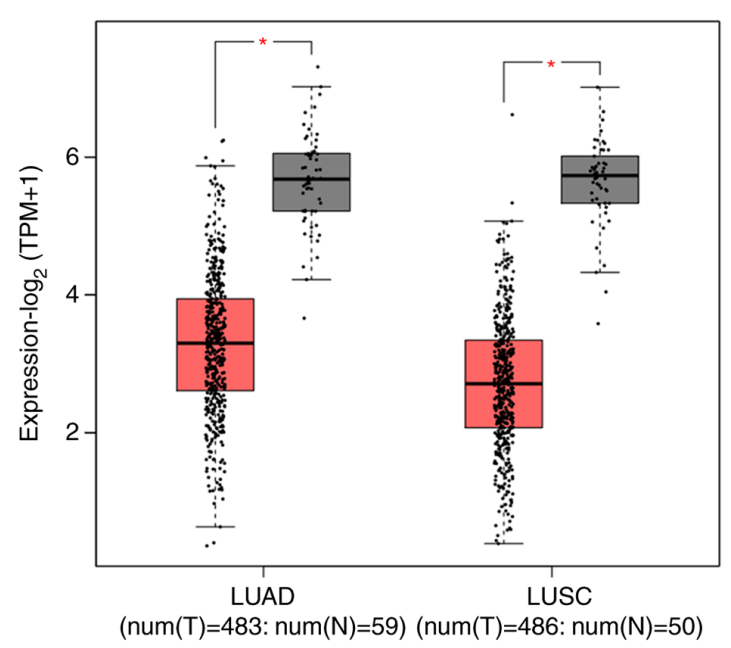

B

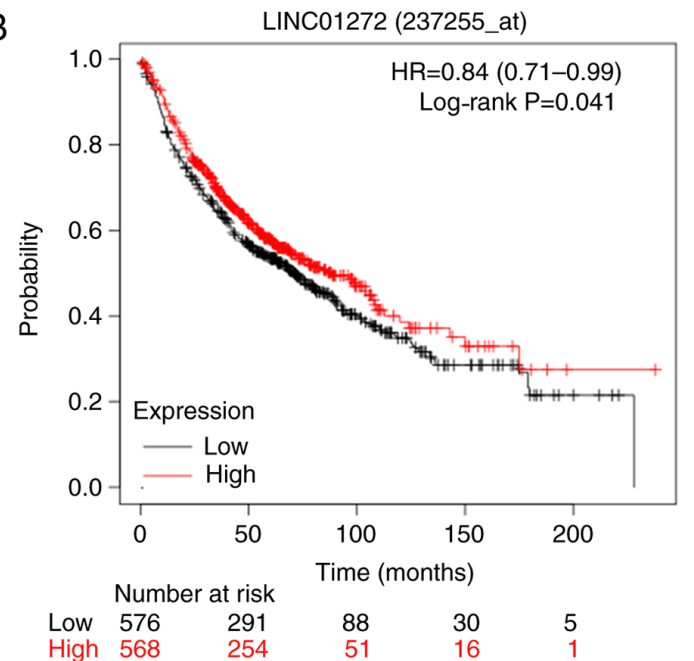

Figure 1. LINC01272 expression and its relationship with overall survival based on bioinformatics analysis. (A) LINC01272 expression in non-small cell lung cancer tumor tissues and normal tissues was determined using TCGA database. ${ }^{*} \mathrm{P}<0.05$ vs. normal. (B) Relationship between LINC01272 expression and overall survival in patients with lung cancer was determined using Gene Expression Omnibus, the European Genome-Phenome Archive and TCGA database (log-rank $\mathrm{P}=0.041)$. LUAD, lung adenocarcinoma; LUSC, lung squamous cell carcinoma; T, tumor; N, normal; TCGA, The Cancer Genome Atlas.

(Beyotime Institute of Biotechnology). NSCLC cells were seeded into 96 well plates at the density of $3 \times 10^{3}$ cells/well and cultured in a humidified incubator at $37^{\circ} \mathrm{C}$. At $0,24,48$ and $72 \mathrm{~h}, \mathrm{CCK}-8$
(10 $\mu \mathrm{l})$ reagent was added to each well and cells were further incubated for $2 \mathrm{~h}$ at $37^{\circ} \mathrm{C}$. The optical density was evaluated at $450 \mathrm{~nm}$ using a microplate analyzer (Bio-Rad Laboratories, Inc.). 

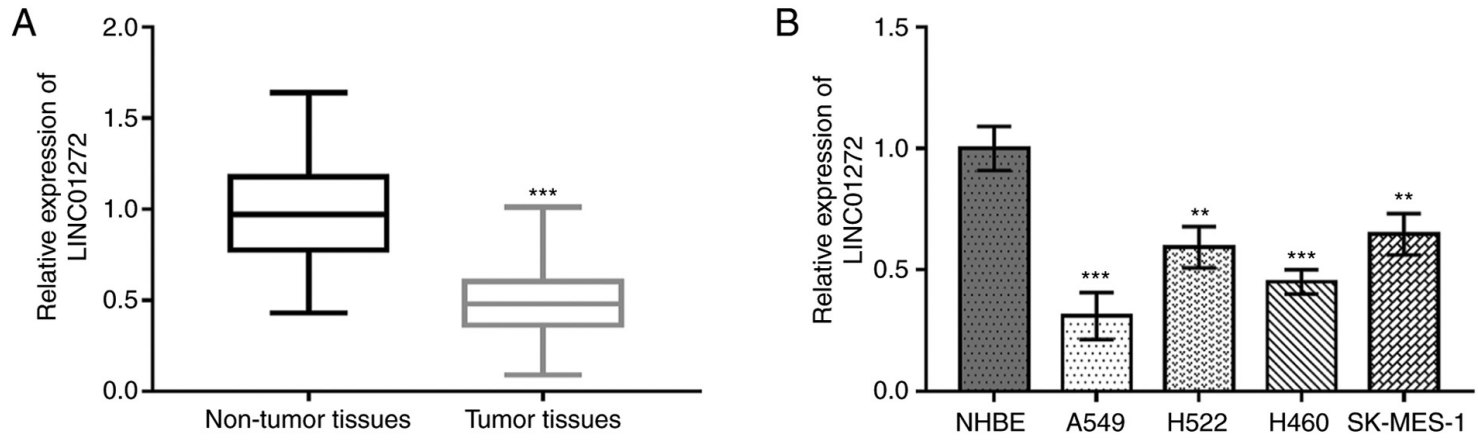

Figure 2. Downregulation of LINC01272 in NSCLC tumor tissues and cell lines. (A) Relative expression of LINC01272 in NSCLC tumor tissues and non-tumor tissues. (B) Relative expression of LINC01272 in NHBE and NSCLC cell lines. ${ }^{* *} \mathrm{P}<0.01$ and ${ }^{* * * *} \mathrm{P}<0.001$ vs. non-tumor tissues or NHBE cells. NSCLC, non-small cell lung cancer.

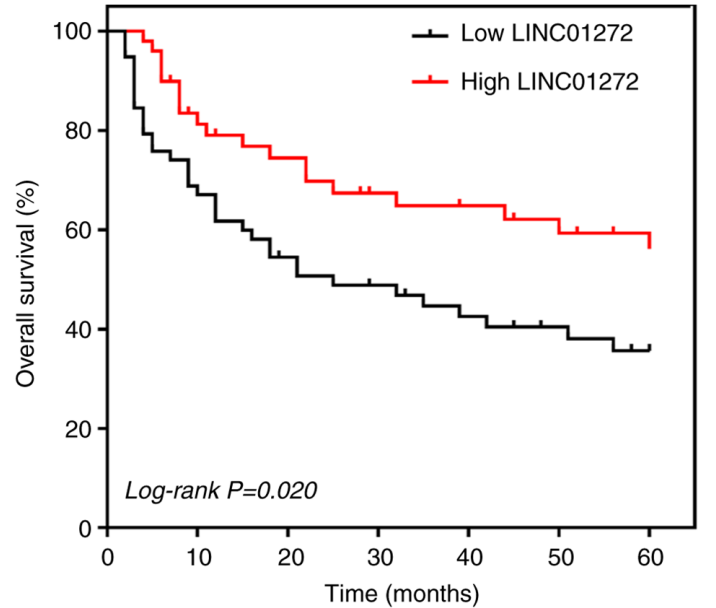

Figure 3. Overall survival of patients with non-small cell lung cancer and low LINC01272 expression was low than that of patients with high LINC01272 expression (log-rank $\mathrm{P}=0.020$ ).

Cell migration and invasion assays. The migratory and invasive abilities of A549 and H460 cells were determined using Transwell chambers (Corning, Inc.). Chambers precoated with Matrigel (Corning, Inc.) were used for invasion assay, whereas chambers not precoated with Matrigel were used for migration assay. A549 and H460 cells ( $3 \times 10^{4}$ cells/well) were seeded into the upper chamber with serum-free DMEM medium, and the lower chamber was filled with DMEM medium supplemented with $10 \%$ FBS. After incubation at $37^{\circ} \mathrm{C}$ for $48 \mathrm{~h}$, cells in the lower chambers were stained with $0.1 \%$ crystal violet for $20 \mathrm{~min}$ at room temperature. The number of migrated or invaded cells in five randomly selected fields was counted under an inverted light microscope (Olympus Corporation; magnification, x200).

Dual-luciferase reporter assay. The binding sequence of LINC01272 to miR-1303 was obtained using DIANA (http://starbase.sysu.edu.cn/) platform. The wild type (WT) LINC01272 sequence containing the miR-1303-binding sequence and the mutant (MUT) LINC01272 sequence were cloned into the pmirGLO dual luciferase miRNA target expression vector (Promega Corporation). Then, LINC01272 (WT) and LINC01272 (MUT) were co-transfected with miR-1303 mimic or mimic NC, respectively, into A549 cells using Lipofectamine 3000 reagent (Invitrogen; Thermo Fisher
Scientific, Inc.). After $48 \mathrm{~h}$ transfection, the relative luciferase activity was measured using the dual-luciferase reporter assay system (Promega Corporation). Firefly luciferase activity was normalized to Renilla luciferase activity. All procedures followed the manufacturers' instructions.

Statistical analysis. Data were presented as the means \pm standard deviation. Differences between two groups were analyzed using paired Student's t-test or $\chi^{2}$ test, and comparisons between multiple groups were assessed using one-way ANOVA followed by Tukey's post hoc test. Kaplan-Meier curves and log-rank test were used to analyze the relationship between LINC01272 expression and the overall survival of patients with NSCLC. The prognostic value of LINC01272 in patients with NSCLC was confirmed by univariate and multivariate Cox regression analysis. Each experiment was repeated at least three times. SPSS 22.0 software (IBM Corp.) and GraphPad Prism 7.0 software (GraphPad Software, Inc.) were used to perform analyses. $\mathrm{P}<0.05$ was considered to indicate a statistically significant difference.

\section{Results}

LINC01272 expression and its relationship with patient overall survival based on bioinformatics analysis. According to bioinformatics analysis, the results from TCGA database analysis revealed that the expression of LINC01272 was downregulated in NSCLC tumors compared with normal tissues (Fig. 1A), and the results from GEO, EGA and TCGA database analyses showed that low LINC01272 expression was associated with worse overall survival in patients with lung cancer (Fig. 1B).

LINC01272 is downregulated in NSCLC tissues and cell lines. The expression level of LINC01272 in NSCLC tissues and cell lines was evaluated using RT-qPCR. As presented in Fig. 2A, LINC01272 expression was significantly decreased in NSCLC tissues compared with adjacent non-tumor tissues $(\mathrm{P}<0.001)$. In addition, LINC01272 expression was significantly decreased in NSCLC cells compared with NHBE cells (all $\mathrm{P}<0.01$; Fig. 2B). Furthermore, because A549 and H460 cells expressed the lowest levels of LINC01272, these cells lines were used for subsequent experiments.

Relationship between LINC01272 expression and the clinicopathological characteristics of patients with NSCLC. Analysis 
Table II. Cox regression analysis results for patients with non-small cell lung cancer.

\begin{tabular}{|c|c|c|c|c|c|c|}
\hline \multirow[b]{2}{*}{ Variables } & \multicolumn{3}{|c|}{ Univariate analysis } & \multicolumn{3}{|c|}{ Multivariate analysis } \\
\hline & HR & $95 \%$ CI & P-value & HR & $95 \%$ CI & P-value \\
\hline Age & 1.365 & $0.815-2.241$ & 0.267 & - & - & - \\
\hline Sex & 1.226 & $0.761-1.960$ & 0.403 & - & - & - \\
\hline Smoking & 1.872 & $0.758-2.955$ & 0.181 & - & - & - \\
\hline Tumor size & 1.376 & $0.862-2.103$ & 0.159 & - & - & - \\
\hline Lymph node metastasis & 1.599 & $1.022-2.389$ & $0.038^{\mathrm{a}}$ & 1.423 & $0.927-2.138$ & 0.091 \\
\hline Differentiation & 1.285 & $1.018-2.654$ & $0.042^{\mathrm{a}}$ & 1.253 & $0.906-1.966$ & 0.128 \\
\hline TNM stage & 1.941 & $1.385-3.159$ & $0.012^{\mathrm{a}}$ & 1.596 & $1.107-2.506$ & $0.027^{\mathrm{a}}$ \\
\hline LINC01272 & 2.085 & $1.523-3.503$ & $0.005^{\mathrm{b}}$ & 1.988 & $1.290-3.078$ & $0.009^{\mathrm{b}}$ \\
\hline
\end{tabular}

${ }^{a} \mathrm{P}<0.05$ and ${ }^{\mathrm{b}} \mathrm{P}<0.01$. TNM, Tumor-Node-Metastasis; CI, confidence interval; HR, hazard ratio.

A

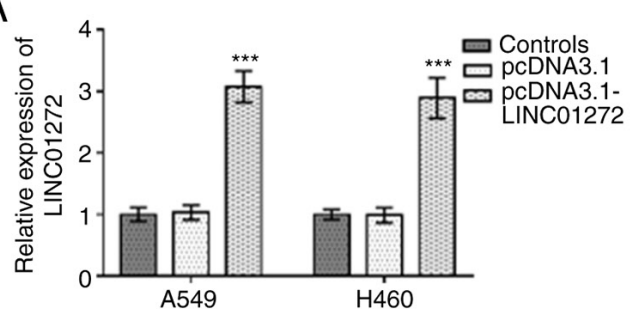

B

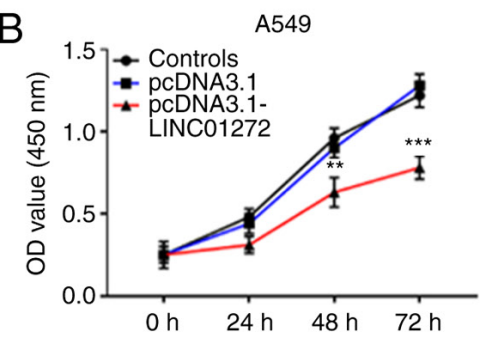

C

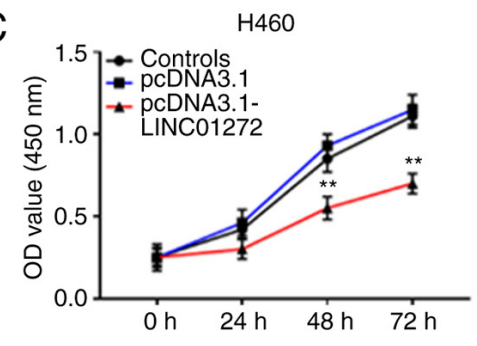

D

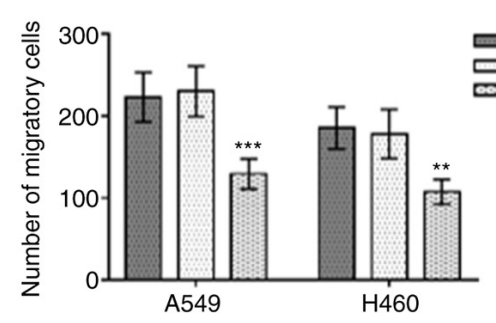

Controls

$\square$ pcDNA3.1

pcDNA3.1-LINC01272

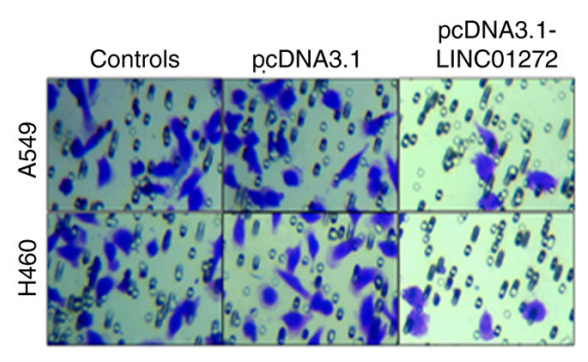

$\mathrm{E}$
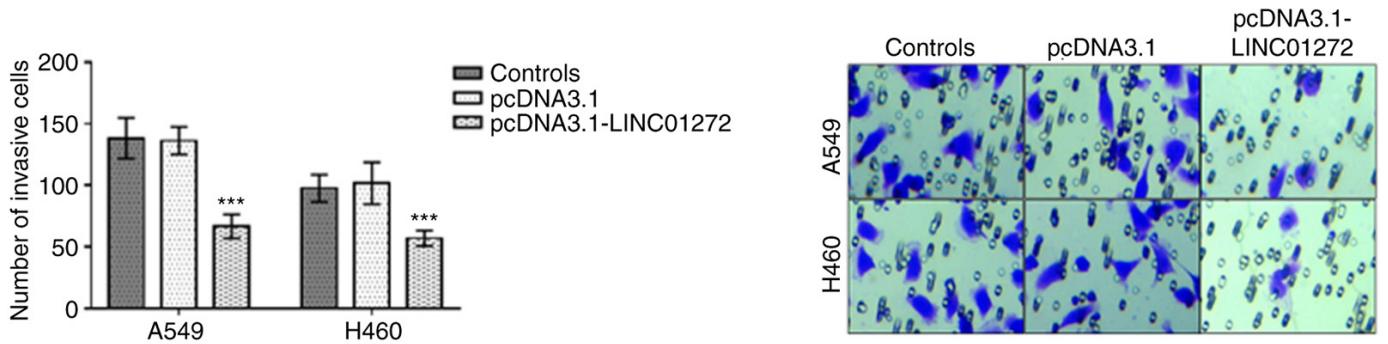

Figure 4. Inhibitory effects of LINC01272 overexpression on non-small cell lung cancer cell proliferation, migration and invasion. (A) Relative expression of LINC01272 was significantly increased after transfection with pcDNA3.1-LINC01272 in A549 cells and H460 cells. Proliferation of (B) A549 cells and (C) H460 cells was significantly inhibited by LINC01272 overexpression. In A549 cells and H460 cells, LINC01272 overexpression decreased the (D) migratory and (E) invasive abilities. ${ }^{* * *} \mathrm{P}<0.01$ and ${ }^{* * *} \mathrm{P}<0.001$ vs. control. OD, optical density.

of the relationship between LINC01272 expression and the clinicopathological characteristics of patients with NSCLC suggested that LINC01272 might be involved in the development of NSCLC. The median expression value of miR-1303 (0.50) was used as the cutoff value to divide the patients into low and high miR-1303 expression groups. As presented in Table I, LINC01272 expression was associated with tumor size $(\mathrm{P}=0.021)$, lymph node metastasis $(\mathrm{P}=0.037)$ and Tumor-Node-Metastasis (TNM) stage $(\mathrm{P}=0.009)$. However, there were no association between LINC01272 expression and the other variables, including age, sex, smoking and differentiation (all $\mathrm{P}>0.05)$.

Association between LINC01272 expression and the overall survival of patients with NSCLC. The prognostic value of LINC01272 expression in patients with NSCLC was evaluated by Kaplan-Meier survival curves and Cox regression analyses. Patients with NSCLC with high LINC01272 expression had 
A

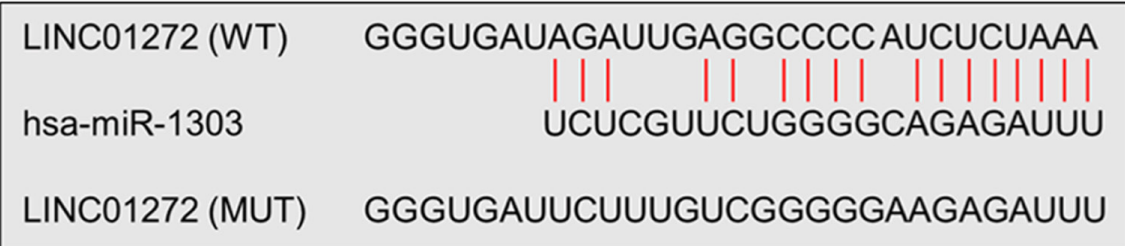

B
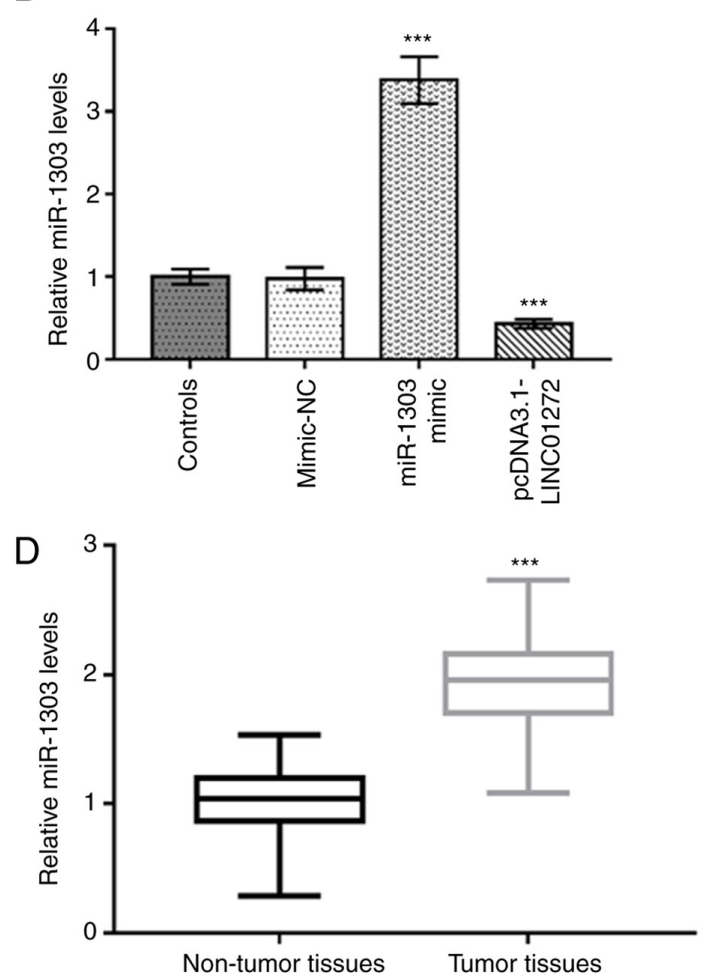

C

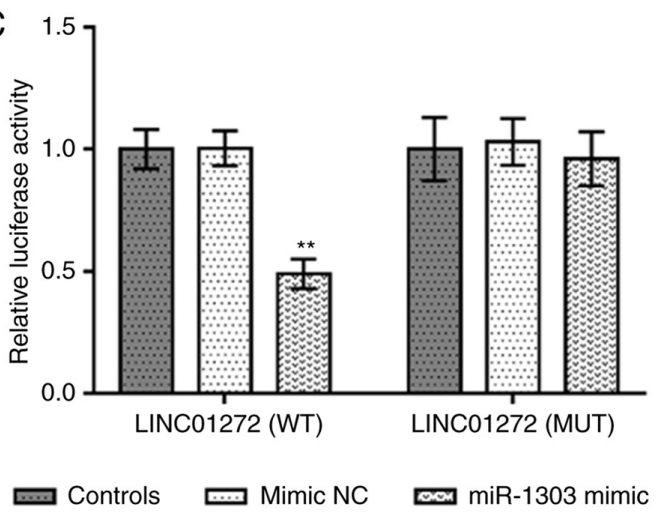

E

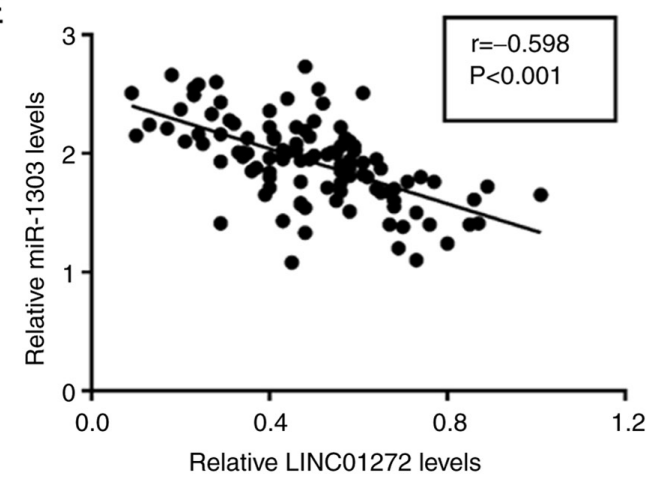

Figure 5. Negative relationship between LINC01272 and miR-1303 in NSCLC. (A) Complementary binding sequences between LINC01272 and miR-1303. (B) Relative miR-1303 expression was upregulated after transfection with miR-1303 mimic, but inhibited following LINC01272 overexpression in A549 cells. (C) Relative luciferase activity in LINC01272 (WT) group was inhibited after miR-1303 overexpression, whereas there was no change in luciferase activity in LINC01272 (MUT) group. (D) Relative miR-1303 levels in tumor tissues and adjacent non-tumor tissues. (E) miR-1303 expression was negatively correlated with LINC01272 expression in NSCLC tissues $(\mathrm{r}=-0.598, \mathrm{P}<0.001) .{ }^{* *} \mathrm{P}<0.01$ and ${ }^{* * *} \mathrm{P}<0.001$ vs. controls or non-tumor tissues. miR, microRNA; NC, negative control; NSCLC, non-small cell lung cancer; WT, wild type; MUT, mutant.

higher overall survival than those with low LINC01272 expression (log-rank $\mathrm{P}=0.020$; Fig. 3). In addition, the results from Cox regression analysis (Table II) indicated that LINC01272 expression, lymph node metastasis, differentiation and TNM stage were associated with the overall survival of patients with NSCLC, and that LINC01272 expression and TNM stage could be used as two independent prognostic factors [LINC01272, hazard ratio $(\mathrm{HR})=1.988,95 \%$ confidence interval $(\mathrm{CI})=1.290-3.078 ; \mathrm{P}=0.009$; TNM stage: $\mathrm{HR}=1.596$, $95 \% \mathrm{CI}=1.107-2.506 ; \mathrm{P}=0.027]$.

Inhibitory effects of LINC01272 overexpression on NSCLC cell proliferation, migration and invasion. Following transfection with pcDNA3.1 or pcDNA3.1-LINC01272, the expression level of LINC01272 was significantly increased by pcDNA3.1-LINC01272 in A549 cells and H460 cells (Fig. 4A; $\mathrm{P}<0.001$ ). Furthermore, the proliferation of A549 cells (Fig. 4B; $\mathrm{P}<0.01$ ) and $\mathrm{H} 460$ cells (Fig. 4C; $\mathrm{P}<0.01$ ) was significantly inhibited following LINC01272 overexpression. In A549 cells and H460 cells, the migratory (Fig. 4D; P<0.01) and invasive (Fig. 4E; $\mathrm{P}<0.001$ ) abilities were significantly inhibited by LINC01272 overexpression.

Negative relationship between LINC01272 and miR-1303 in NSCLC. The complementary binding sequences between LINC01272 and miR-1303 were seen in Fig. 5A. As presented in Fig. 5B, miR-1303 was significantly upregulated following transfection with miR-1303 mimic but downregulated after LINC01272 overexpression in A549 cells $(\mathrm{P}<0.001)$. Furthermore, a dual-luciferase reporter assay was conducted to confirm the interaction between LINC01272 and miR-1303 in NSCLC cells. The results demonstrated that overexpression of miR-1303 inhibited the relative luciferase activity of LINC01272 (WT) group $(\mathrm{P}<0.01)$, whereas no changes were observed in the luciferase activity of LINC01272 (MUT) group ( $\mathrm{P}>0.05$; Fig. 5C). As presented in Fig. 5D, miR-1303 expression was significantly increased in tumor tissues compared with adjacent non-tumor tissues $(\mathrm{P}<0.001)$. In addition, a negative correlation was observed between miR-1303 expression and LINC01272 expression in NSCLC tissues (Fig. 5E; r=-0.598, P<0.001). 
A

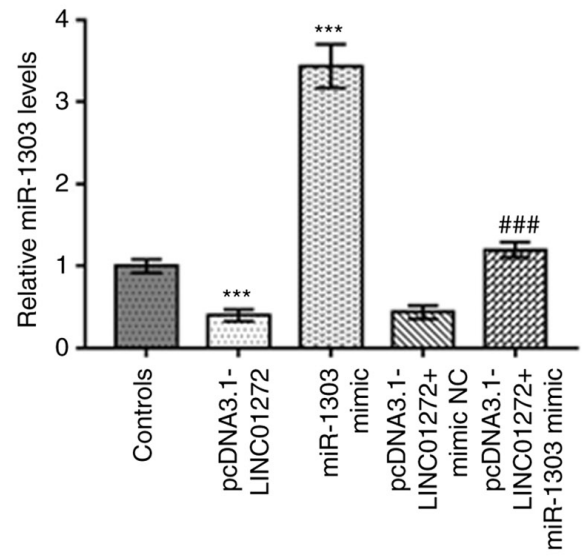

C

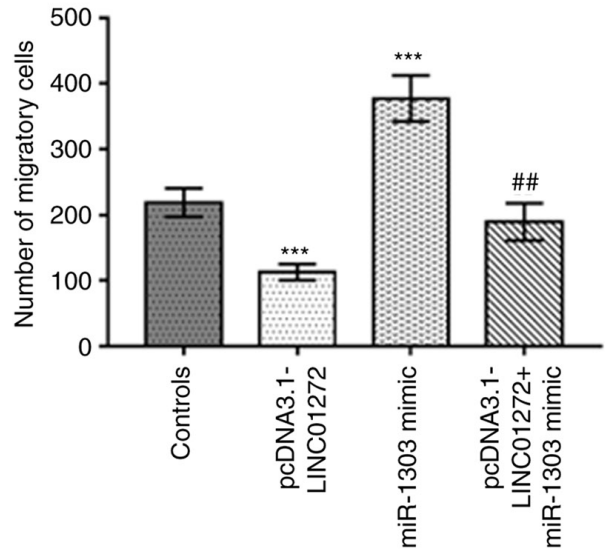

$\mathrm{D}$

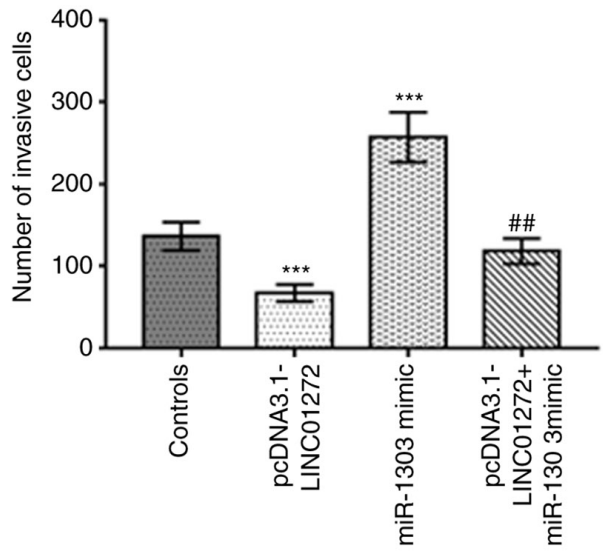

B
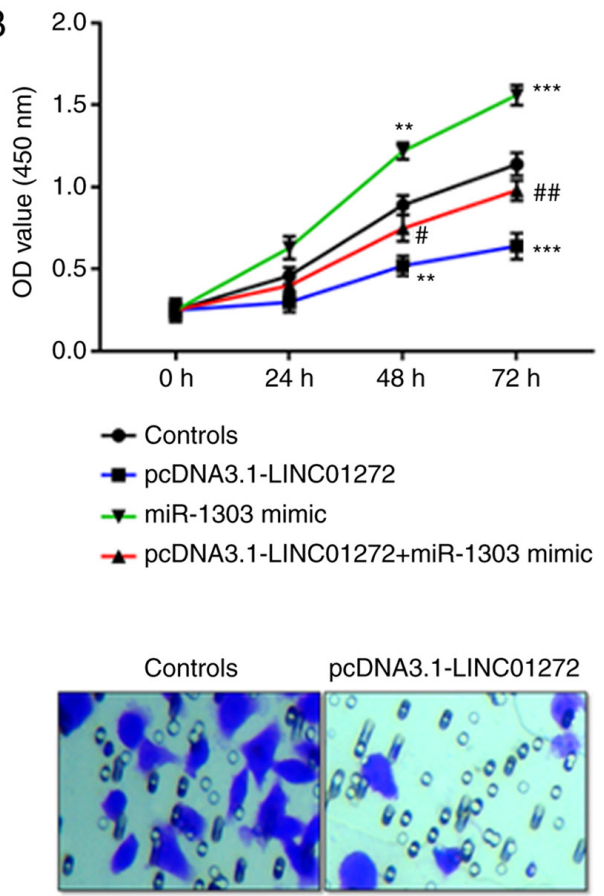

pcDNA3.1-LINC01272+

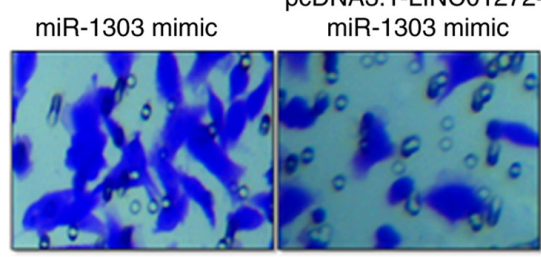

Controls

pcDNA3.1-LINC01272
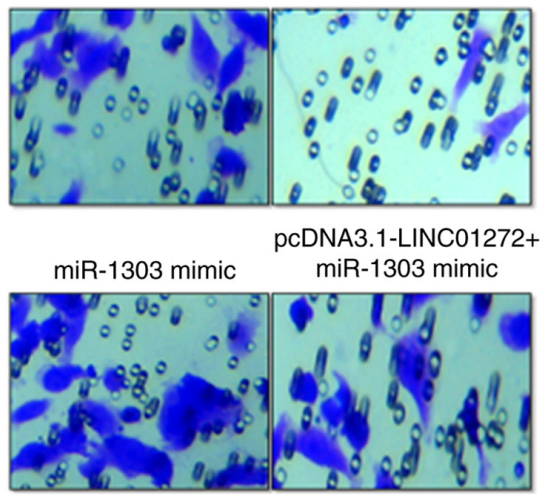

Figure 6. miR-1303 overexpression reverses the function of LINC01272 in NSCLC cells. (A) miR-1303 mimic reversed the inhibitory effect of pcDNA3.1-LINC01272 on miR-1303 expression in NSCLC cells. Overexpression of miR-1303 reversed the inhibitory effects of LINC01272 overexpression on NSCLC (B) cell proliferation, (C) migration and (D) invasion. ${ }^{* * *} \mathrm{P}<0.01$ and ${ }^{* * * *} \mathrm{P}<0.001$ vs. controls. ${ }^{\#} \mathrm{P}<0.05,{ }^{\# \#} \mathrm{P}<0.01$ and ${ }^{\# \# \#} \mathrm{P}<0.001$ vs. pcDNA3.1-LINC01272. OD, optical density; miR, microRNA; NSCLC, non-small cell lung cancer.

miR-1303 overexpression reverses the function of LINC01272 in NSCLC cells. As presented in Fig. 6A, miR-1303 expression was significantly decreased after transfection with pcDNA3.1-LINC01272, whereas it was significantly increased by miR-1303 mimic in A549 cells (all $\mathrm{P}<0.001$ ). In addition, miR-1303 mimic reversed the suppressive effect of pcDNA3.1-LINC01272 on the expression of miR-1303 in A549 cells $(\mathrm{P}<0.001)$. Overexpression of LINC01272 inhibited, while miR-1303 overexpression promoted the proliferation, migration and invasion of A549 cells (Fig. 6B-D; $\mathrm{P}<0.01$ ).
Furthermore, miR-1303 overexpression reversed the inhibitory effects of LINC01272 overexpression on the function of A549 cells (Fig. 6B-D; $\mathrm{P}<0.05$ ).

\section{Discussion}

It has been demonstrated that lncRNAs aberrant expression is closely related to the progression of numerous types of cancer $(6,7,21)$, suggesting that lncRNAs might be involved in the progression of cancer. In addition, numerous lncRNAs, 
including PTAR (22), NBR2 (23) and DLEU2 (24), have been reported to be abnormally expressed in NSCLC. According to bioinformatics analysis of data from TCGA database, LINC01272 expression level was found to be significantly downregulated in NSCLC. The findings from the present study confirmed the downregulation of LINC01272 in NSCLC tumor tissues and cells. Furthermore, LINC01272 expression was significantly correlated with tumor size, lymph node metastasis and TNM stage in patients with NSCLC. In addition, LINC01272 has been found to serve as an important regulator in other diseases. For example, LINC01272 is upregulated in gastric cancer and its expression is associated with tumor stage and lymph node metastasis (9). Wang et al (10) reported an increased expression of LINC01272 in tissues and plasma samples of patients with inflammatory bowel disease compared with healthy volunteers. The present study hypothesized therefore that LINC01272 may be involved in the progression of NSCLC.

Increasing evidence has demonstrated that certain IncRNAs can function as potential biomarkers for NSCLC prognosis (25), such as SLC16A1-AS1 (26), XIST (27) and LINC-PINT (28). Our preliminary bioinformatics analysis using GEO, EGA and TCGA database data reported that patients with lung cancer and with low LINC01272 expression had a significantly worse overall survival than patients with high LINC01272 expression. Furthermore, in the present study, decreased expression of LINC01272 was found in NSCLC tissues. The present study analyzed the prognostic significance of LINC01272 in NSCLC. It has been reported that SLC16A1-AS1 $(\mathrm{HR}=3.351$, 95\% $\mathrm{CI}=2.027-5.541, \mathrm{P}<0.001)(26)$, XIST $(\mathrm{HR}=2.645$, 95\% $\mathrm{CI}=1.672-7.393, \mathrm{P}=0.029)(27)$ and LINC-PINT $(\mathrm{HR}=2.628,95 \% \mathrm{CI}=1.589-4.348, \mathrm{P}<0.001)(28)$ can be used as prognostic biomarkers in patients with NSCL. The prognostic value of LINC01272 was evaluated according to the 5-year survival information of patients with NSCLC in the present study. The overall survival of patients with low LINC01272 expression was worse compared with that of patients with high LINC01272 expression. In addition, LINC01272 was independently related to overall survival, suggesting that LINC01272 may be considered as a potential prognostic biomarker for NSCLC. Since the HR of LINC01272 is lower than the HRs of SLC16A1-AS1, XIST and LINC-PINT, the overall mortality risk of patients with NSCLC and with low LINC01272 expression was relatively low.

Increasing evidence indicates that $\operatorname{lncRNAs}$ can regulate NSCLC tumor biological functions (29-31). For example, LINC00673 can regulate the proliferation, migration and invasion of NSCLC cells by sponging miR-150-5p (29). Furthermore, PCAT7 can promote the proliferation, migration and invasion, and inhibit the apoptosis of NSCLC cells by inhibiting miR-134-5p (30). A study by An et al (31) reported that LINC00668 downregulation inhibits NSCLC cell proliferation, migration and invasion, and stimulates NSCLC cell apoptosis. The results from the present study demonstrated that LINC01272 overexpression could inhibit NSCLC cell proliferation, migration and invasion. In addition, LINC01272 knockdown has been found to inhibit the biological function of gastric cancer cells (9). LINC01272 may therefore serve an anticancer role in NSCLC.
Previous studies have demonstrated that miRNA-lncRNA interactions play important roles in the occurrence and development of cancer (32-34). According to bioinformatics prediction, miR-1303 was identified as a target gene of LINC01272 in the present study, and this interaction was confirmed through a luciferase reporter assay. miR-1303 is a key molecule involved in various cancers, such as prostate cancer (15) and gastric cancer (16). The present study reported a higher miR-1303 expression in tumor tissues compared with non-tumor tissues, which was in accordance with a study from Chen et al (35). Furthermore, miR-1303 expression was negatively correlated with LINC01272 expression in tumor tissues of patients with NSCLC. In addition, overexpression of LINC01272 inhibited miR-1303 expression in NSCLC cells, and miR-1303 overexpression reversed the effects of LINC01272 on NSCLC cell proliferation, migration and invasion, suggesting that LINC01272 may inhibit NSCLC cell function by targeting miR-1303. Certain IncRNAs have been found to exert their biological functions by regulating miR-1303 in several diseases. For example, BCRT1 was reported to contribute to breast cancer cell proliferation, migration and invasion by targeting miR-1303/PTBP3 axis (36). In addition, LINC01433 upregulation can promote cell proliferation and migration by sponging miR-1301 in esophageal squamous cell carcinoma (37).

A study by Liu et al (15) reported that miR-1303 can enhance the cell proliferation, migration and invasion via targeting DKK3 in prostate cancer. Cheng et al (38) demonstrated that miR-1303-p can regulate the cell proliferation and apoptosis of clear cell renal cell carcinoma by targeting STARD9. Therefore, we speculated that miR-1303 may regulate NSCLC cell function by targeting DKK3 or STARD. However, whether DKK3 and STARD9 are targets of miR-1303 in NSCLC remains unclear. Thus, the lack of miR-1303 target is a major limitation of the present study, and further investigation is therefore required to validate the results. In addition, the sample size was small, which is also a limitation of this study, and future study including a large research cohort is needed.

In conclusion, the present study demonstrated that LINC01272 expression was decreased in NSCLC tumor tissues and NSCLC cells compared with normal tissues and cells, suggesting that LINC01272 may serve as an independent prognostic biomarker for patients with NSCLC. In addition, LINC01272 overexpression could inhibit NSCLC cell proliferation, migration and invasion by inhibiting miR-1303 in NSCLC. The novel LINC01272 /miR-1303 axis may therefore be considered as a new biomarker and therapeutic target for NSCLC treatment.

\section{Acknowledgements}

Not applicable.

\section{Funding}

No funding was received.

\section{Availability of data and materials}

The datasets used and/or analyzed during the current study are available from the corresponding author on reasonable request. 


\section{Authors' contributions}

SZ and JZ designed the study, performed clinical studies and analyzed data. JZ performed the cell experiments. SZ and JZ wrote and revised the manuscript. SZ and JZ confirmed the authenticity of all raw data. All authors have read and approved the final manuscript.

\section{Ethics approval and consent to participate}

A signed written informed consent was obtained from each patient and the experimental procedures were all in accordance with the guideline of the Ethics Committee of Weifang People's Hospital (approval no. 011827).

\section{Patient consent for publication}

Not applicable.

\section{Competing interests}

The authors declare that they have no competing interests.

\section{References}

1. Bray F, Ferlay J, Soerjomataram I, Siegel RL, Torre LA and Jemal A: Global cancer statistics 2018: GLOBOCAN estimates of incidence and mortality worldwide for 36 cancers in 185 countries. CA Cancer J Clin 68: 394-424, 2018.

2. Goebel C, Louden CL, McKenna R Jr, Onugha O, Wachtel A and Long T: Diagnosis of non-small cell lung cancer for early stage asymptomatic patients. Cancer Genomics Proteomics 16 : 229-244, 2019

3. Yang Q, Tang Y, Tang C, Cong H, Wang X, Shen X and Ju S: Diminished LINC00173 expression induced miR-182-5p accumulation promotes cell proliferation, migration and apoptosis inhibition via AGER/NF- $\kappa$ B pathway in non-small-cell lung cancer. Am J Transl Res 11: 4248-4262, 2019.

4. Wang J, Su Z, Lu S, Fu W, Liu Z, Jiang X and Tai S: LncRNA HOXA-AS2 and its molecular mechanisms in human cancer. Clin Chim Acta 485: 229-233, 2018.

5. Chi Y, Wang D, Wang J, Yu W and Yang J: Long non-coding RNA in the pathogenesis of cancers. Cells 8: 1015, 2019.

6. Hu S, Zheng Q, Xiong J, Wu H, Wang W and Zhou W: Long non-coding RNA MVIH promotes cell proliferation, migration, invasion through regulating multiple cancer-related pathways, and correlates with worse prognosis in pancreatic ductal adenocarcinomas. Am J Transl Res 12: 2118-2135, 2020.

7. Wang Y, Ding X, Hu H, He Y, Lu Z, Wu P, Tian L, Xia T, Yin J, Yuan $\mathrm{H}$, et al: Long non-coding RNA lnc-PCTST predicts prognosis through inhibiting progression of pancreatic cancer by downregulation of TACC-3. Int J Cancer 143: 3143-3154, 2018.

8. Liu XH, Sun M, Nie FQ, Ge YB, Zhang EB, Yin DD, Kong R, Xia R, Lu KH, Li JH, et al: Lnc RNA HOTAIR functions as a competing endogenous RNA to regulate HER2 expression by sponging miR-331-3p in gastric cancer. Mol Cancer 13: 92 , 2014.

9. Leng X, Liu G, Wang S, Song J, Zhang W, Zhang X, Rong L, $\mathrm{Ma}$ Y and Song F: LINC01272 promotes migration and invasion of gastric cancer cells via EMT. Onco Targets Ther 13 3401-3410, 2020.

10. Wang S, Hou Y, Chen W, Wang J, Xie W, Zhang X and Zeng L: KIF9-AS1, LINC01272 and DIO3OS 1ncRNAs as novel biomarkers for inflammatory bowel disease. Mol Med Rep 17: 2195-2202, 2018.

11. Hung J, Scanlon JP, Mahmoud AD, Rodor J, Ballantyne M, Fontaine MAC, Temmerman L, Kaczynski J, Connor KL, Bhushan R, et al: Novel plaque enriched long noncoding RNA in atherosclerotic macrophage regulation (PELATON). Arterioscler Thromb Vasc Biol 40: 697-713, 2020.
12. Wang Z, Sha HH and Li HJ: Functions and mechanisms of miR-186 in human cancer. Biomed Pharmacother 119: 109428, 2019.

13. Iacona JR and Lutz CS: miR-146a-5p: Expression, regulation, and functions in cancer. Wiley Interdiscip Rev RNA 10: e1533, 2019.

14. Mamoori A, Gopalan V and Lam AK: Role of miR-193a in cancer: Complexity and factors control the pattern of its expression. Curr Cancer Drug Targets 18: 618-628, 2018.

15. Liu B, Zhou W, Jiang H, Xiang Z and Wang L: miR-1303 promotes the proliferation, migration and invasion of prostate cancer cells through regulating the Wnt $/ \beta$-catenin pathway by targeting DKK3. Exp Ther Med 18: 4747-4757, 2019.

16. Zhang SJ, Feng JF, Wang L, Guo W, Du YW, Ming L and Zhao GQ: miR-1303 targets claudin-18 gene to modulate proliferation and invasion of gastric cancer cells. Dig Dis Sci 59: 1754-1763, 2014

17. Lv QL, Wang LC, Li DC, Lin QX, Shen XL, Liu HY, Li M, Ji YL, Qin CZ and Chen SH: Knockdown lncRNA DLEU1 inhibits gliomas progression and promotes temozolomide chemosensitivity by regulating autophagy. Front Pharmacol 11: 560543, 2020.

18. Tang Z, Li C, Kang B, Gao G, Li C and Zhang Z: GEPIA: A web server for cancer and normal gene expression profiling and interactive analyses. Nucleic Acids Res 45 (W1): W98-W102, 2017.

19. Győrffy B, Surowiak P, Budczies J and Lánczky A: Online survival analysis software to assess the prognostic value of biomarkers using transcriptomic data in non-small-cell lung cancer. PLoS One 8: e82241, 2013.

20. Schmittgen TD and Livak KJ: Analyzing real-time PCR data by the comparative C(T) method. Nat Protoc 3: 1101-1108, 2008

21. Feng J, Li J, Qie P, Li Z, Xu Y and Tian Z: Long non-coding RNA (lncRNA) PGM5P4-AS1 inhibits lung cancer progression by up-regulating leucine zipper tumor suppressor (LZTS3) through sponging microRNA miR-1275. Bioengineered 12: 196-207, 2021.

22. Yu W, Sun Z, Yang L, Han Y, Yue L, Deng L and Yao R: lncRNA PTAR promotes NSCLC cell proliferation, migration and invasion by sponging microRNA-101. Mol Med Rep 20: 4168-4174, 2019.

23. Gao YP, Li Y, Li HJ and Zhao B: LncRNA NBR2 inhibits EMT progression by regulating Notch1 pathway in NSCLC. Eur Rev Med Pharmacol Sci 23: 7950-7958, 2019.

24. Zhou Y, Shi H, Du Y, Zhao G, Wang X, Li Q, Liu J, Ye L, Shen Z, Guo Y and Huang Y: lncRNA DLEU2 modulates cell proliferation and invasion of non-small cell lung cancer by regulating miR-30c-5p/SOX9 axis. Aging (Albany NY) 11: 7386-7401, 2019.

25. Song JY, Li XP, Qin XJ, Zhang JD, Zhao JY and Wang R: A fourteen-lncRNA risk score system for prognostic prediction of patients with non-small cell lung cancer. Cancer Biomark 29: 493-508, 2020

26. Liu HY, Lu SR, Guo ZH, Zhang ZS, Ye X, Du Q, Li H, Wu Q Yu B, Zhai Q and Liu JL: IncRNA SLC16A1-AS1 as a novel prognostic biomarker in non-small cell lung cancer. J Investig Med 68: 52-59, 2020.

27. Fang H, Yang L, Fan Y, Mo C, Luo L, Liang D and Jiang Y: Upregulation of tissue long noncoding RNA X inactive specific transcript predicts poor postoperative survival in patients with non-small cell lung cancer. Medicine (Baltimore) 99: e21789, 2020.

28. Zhang C, Gong C, Li J and Tang J: Downregulation of long non-coding RNA LINC-PINT serves as a diagnostic and prognostic biomarker in patients with non-small cell lung cancer. Oncol Lett 21: 210, 2021.

29. Lu W, Zhang H, Niu Y, Wu Y, Sun W, Li H, Kong J, Ding K, Shen $\mathrm{HM}$, Wu $\mathrm{H}$, et al: Long non-coding RNA linc00673 regulated non-small cell lung cancer proliferation, migration, invasion and epithelial mesenchymal transition by sponging miR-150-5p. Mol Cancer 16: 118, 2017.

30. Liu Q, Wu Y, Xiao J and Zou J: Long non-coding RNA prostate cancer-associated transcript 7 (PCAT7) induces poor prognosis and promotes tumorigenesis by inhibiting mir-134-5p in non-small-cell lung (NSCLC). Med Sci Monit 23: 6089-6098, 2017.

31. An YX, Shang YJ, Xu ZW, Zhang QC, Wang Z, Xuan WX and Zhang XJ: STAT3-induced long noncoding RNA LINC00668 promotes migration and invasion of non-small cell lung cancer via the miR-193a/KLF7 axis. Biomed Pharmacother 116: $109023,2019$.

32. Luo H, Xu C, Le W, Ge B and Wang T: lncRNA CASC11 promotes cancer cell proliferation in bladder cancer through miRNA-150. J Cell Biochem 120: 13487-13493, 2019. 
33. Luan X and Wang Y: LncRNA XLOC 006390 facilitates cervical cancer tumorigenesis and metastasis as a ceRNA against miR-331-3p and miR-338-3p. J Gynecol Oncol 29: e95, 2018.

34. Zhao W, Geng D, Li S, Chen Z and Sun M: LncRNA HOTAIR influences cell growth, migration, invasion, and apoptosis via the miR-20a-5p/HMGA2 axis in breast cancer. Cancer Med 7 : 842-855, 2018.

35. Chen J, Jiang T, Yu B, Li T, Zhao P, Yuan L and Qi J: Upregulation of microRNA-1303 is a potential prognostic marker of non-small cell lung cancer. Cancer Biomark 28: 439-446, 2020.

36. Liang Y, Song X, Li Y, Chen B, Zhao W, Wang L, Zhang H, Liu Y, Han D, Zhang N, et al: LncRNA BCRT1 promotes breast cancer progression by targeting miR-1303/PTBP3 axis. Mol Cancer 19: 85, 2020.
37. Zheng L, Liu YT, Wu CP, Jiang JT, Zhang L, Wang ZL and Wang QY: Long non-coding RNA linc01433 promotes tumorigenesis and progression in esophageal squamous cell carcinoma by sponging miR-1301. Eur Rev Med Pharmacol Sci 24: 4785-4792, 2020.

38. Cheng T, Shuang W, Ye D, Zhang W, Yang Z, Fang W, Xu H, $\mathrm{Gu}$ M, Xu W and Guan C: SNHG16 promotes cell proliferation and inhibits cell apoptosis via regulation of the miR-1303-p/STARD9 axis in clear cell renal cell carcinoma. Cell Signal 84: 110013, 2021.

c) (i) $\Theta$ This work is licensed under a Creative Common Attribution-NonCommercial-NoDerivatives 4.0 International (CC BY-NC-ND 4.0) License. 